\title{
A LABORATORY APPARATUS FOR THE CONTINUOUS EXTRACTION OF LIQUIDS BY LOW BOILING SOL- VENTS ${ }^{1}$
}

\author{
By R. T. Leslie ${ }^{2}$
}

ABSTRACT

A laboratory apparatus for the extraction of liquids by low-boiling solvents is described. The apparatus is counter-current and continuous in operation. Important details of constructions are given and the method of operation is described. A brief discussion of the performance of the apparatus for the extraction of petroleum fractions with liquid sulphur dioxide is given.

\section{CONTENTS}

I. Introduction

591

II. Construction of glass apparatus

III. Operation of the glass apparatus.

IV. Construction of metal apparatus. 596

V. Operation of the metal apparatus... 597

VI. Performance of the apparatus. 599

VII. Acknowledgment.

\section{INTRODUCTION}

Differences in solubility in liquid sulphur dioxide have been used,,$^{34}$ as a means of separating aromatic and olefinic hydrocarbons from the paraffinic and naphthenic groups. In connection with the problem of determining the composition of petroleum it was deemed advisable to investigate the general method of fractionation by extraction with low-boiling solvents. It was found that extraction has the disadvantage that the distribution coefficients are seldom favorable for the complete separation of a distinct component from a mixture. Attempts have been made to estimate the composition of petroleum by applying correction factors to the sulphur dioxide soluble content of various distillation fractions, but the method is rendered somewhat uncertain by the effect of unknown constituents on the distribution coefficients. ${ }^{56}$ Extraction should be valuable, however, as a method of breaking up the constant boiling mixtures encountered in fractional distillation, and may be supplemented by subsequent further distillation or crystallization, or both, to obtain pure materials.

Sulphur dioxide is one of the best known extractants which has the property of selectively dissolving certain types of hydrocarbons, and considerable technic has been developed for its use both on a commercial and on a laboratory scale. ${ }^{789}$ After some consideration, however,

1 Financial assistance has been received from the research fund of the American Petroleum Institute. This work is part of project No. 6, The Separation, Identification, and Determination of the Constituents of Petroleum.

2 American Petroleum Institute research associate.

P. Walden, Ber., vol. 32, p. 2862, 1899.

4 Edeleanu, Am. Inst. Mining Eng., vol. 93, p. 2313, 1914.

S. E. Bowrey, Pet. Rev., vol. 36, pp. 352, 385, 401, 1917.

- See footnote 4 .

7 See footnote 4.

- Laboratory apparatus described in a circular published by Allgemeine Gesellschaft für Chemische Industrie, M. B. H., Berlin, W8.

R. L. Brandt, Ind. Eng. Chem., vol. 22, p. 218, 1930. 
it was decided that improvement could be made in the laboratory apparatus.

The apparatus which has been developed, although intended primarily for use with sulphur dioxide, can be used with any low-boiling solvent, the density of which is greater than that of the material to be extracted. For use with solvents of densities less than that of the hydrocarbon, some modification is necessary, but the same principles can be used.

Two forms of the apparatus will be described. The first type is entirely of glass and is the simpler to construct, but must be operated at temperatures below the boiling point of sulphur dioxide. The second type is of metal and may be operated at temperatures above the normal boiling point of sulphur dioxide. It also possesses the convenience that the sulphur dioxide need not be removed when the apparatus is not in use.

Both types operate on essentially the same principles, of which the salient features are (1) countercurrent flow of the liquids, (2) maintenance of a considerable excess of sulphur dioxide, (3) automatic control of the height of the column of sulphur dioxide by an overflow tube requiring no valve regulation, and (4) continuous operation in regard to circulation of both sulphur dioxide and hydrocarbon.

\section{CONSTRUCTION OF GLASS APPARATUS}

Figure 1 is nearly self-explanatory, but a few details of construction will be emphasized.

The extraction column, $G$, of the apparatus used in this laboratory is about $80 \mathrm{~cm}$ in height and $1.8 \mathrm{~cm}$ in diameter. It is difficult to maintain a smooth countercurrent flow in columns of smaller diameter. No packing is used because the hydrocarbon tends to cling to it and to displace the sulphur dioxide instead of rising through it. The tube, $O$, is made of $1 \mathrm{~mm}$ capillary to reduce the volume of sulphur dioxide outside the cooling bath and at the same time to afford mechanical support to the column. Smaller capillaries tend to clog. The upper section of the column, $F$, is made of smaller tubing to decrease the volume of immiscible hydrocarbon held up in this part of the apparatus.

The overflow tube, $H$, must be of such a height that the weight of extract in it will more than counterbalance the weight of extract plus the weight of the supernatant hydrocarbon in the column. For two liquids which differ as much in density as sulphur dioxide and petroleum hydrocarbon, it is necessary only to keep the height of the overflow a few centimeters (10 to $15 \mathrm{~cm}$ ) below the outlet tube for the immiscible material. The vent tube $E_{2}$ prevents the overflow tube from acting as a siphon.

The jacket surrounding the column consists of a $5.5 \mathrm{~cm}$ glass tube in which the column is held in place by the split cork at the bottom. This cork is made liquid-tight by pouring a layer of collodion into the bottom of the jacket after it is assembled. Heavy felt packing may be used for heat insulation.

The two extract boilers, $R_{3}$ and $R_{4}$, are made from one-fourth-liter flasks. The reentrant tubes are bent so that they do not open directly over the drain tubes because the latter act as points of ebullition for the boiling sulphur dioxide and would interfere with the ready flow of 
liquid down the reentrant tubes. Individual gas tubes, $M$, should be run from the boilers to the condenser, $C$, for two reasons: First, because the short connecting tube, which results if the two boilers are connected to a single gas tube, allows the sulphur dioxide to distill directly from the hot boiler to the cold one without reaching condenser, $C$, and second, because two legs of the small triangle of glass tubes which is thus formed vary so greatly in temperature when the $\mathrm{SO}_{2}$-cycle is reversed that even Pyrex laboratory glass is likely to crack. The latter difficulty could be obviated by using a spiraled gas connection between the boilers, but the double tube as shown avoids both difficulties. Occasional drying of the gas by passage through calcium chloride is sufficient so that a shunted drying tube is placed in only one of the gas lines.

The trap form of condenser, $C$, was adopted as most satisfactory after several forms of spiral condensers were tried. Considerable cooling agent is consumed at this point so that the jacket, $D$, should be of ample capacity. A 2-liter bottle with the bottom cut off, mounted in an inverted position as shown, was found satisfactory.

The reservoir, $R_{1}$, and the receiver, $R_{2}$, are each of one-half liter capacity. The rise in the delivery tube which connects the column, $F$, with the receiver, $R_{2}$, is convenient to prevent sulphur dioxide from running into the receiver when for reasons to be explained later it is poured into the column at $F$. The vent, $E_{3}$, is necessary to prevent the liquid from flowing irregularly as a result of a slight tendency to siphon over the rise.

To keep the apparatus compact it is much more desirable to force the hydrocarbon in by means of gas pressure at $A$ than to mount the reservoir at a sufficient height for the material to flow in by gravity.

Since very little lubricant can be used on the stopcocks, they should be carefully selected for smoothness and fit.

The heaters on the boilers, $R_{3}$ and $R_{4}$, may be made with about 12 feet of No. 26 B. \& S. gage "Chromel" wire imbedded in "Alundum cement." The heater on $R_{2}$ should have about twice this resistance. These specifications are given for 110 -volt supplies.

The whole apparatus may be mounted on a tall ring stand or a rigid frame in such a way that it forms an easily transportable unit.

\section{OPERATION OF THE GLASS APPARATUS}

Considerable time will be saved in working with samples of unknown composition by a few preliminary experiments with a simple apparatus such as shown in Figure 2. This apparatus is cooled to about $-20^{\circ}$ or $-30^{\circ} \mathrm{C}$. in a bath contained in a liter Dewar tube. The hydrocarbon and sulphur dioxide are mixed in $A$ with the capillary closed by the glass rod. When the layers have separated, the apparatus is lifted out of the bath long enough to permit the removal of the extract layer into $E$. After a precautionary cooling in the bath it is again lifted out and the extract forced out into a cold test tube through $F$ by blowing into $D$. The sulphur dioxide can be boiled out of the extract, collected in a trap, and used for a second extraction. In this way the feasibility of extraction can be determined on a sample of a few milliliters. 
If extraction is practicable it is necessary (1) that this test result

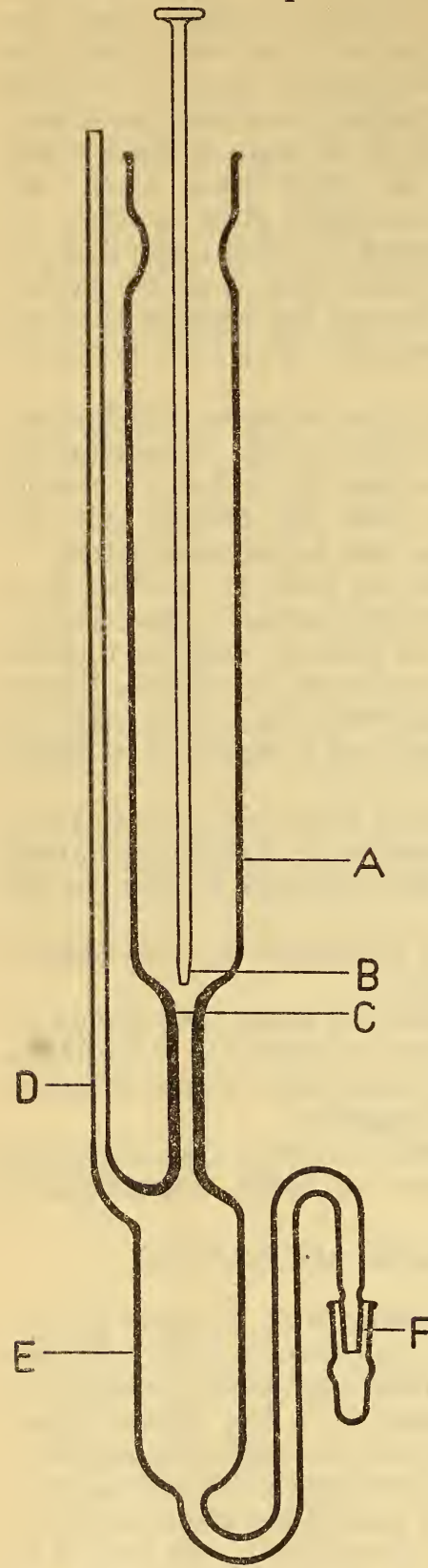

Figdre 2.-Apparatus for test extractions

$A$, chamber in which $\mathrm{SO}_{2}$ and hydrocarbon are mixed; $B$, glass rod with end tapered to fit $C ; C$, capillary tube; $D$, vent tube; $E$, chamber into which lower immiscible layer is drained; $F$, exit tube for liquid (closed with ground stopper). in the formation of two layers, (2) that the hydrocarbon in the two layers differ in composition, (3) that no solid separate at the temperature of extraction. This third condition is necessary since the operation of the large apparatus depends on the flow of cold liquids through small tubes. If the mixture contains a constituent, such as benzene, in sufficient concentration to freeze out at a temperature approaching the boiling point of sulphur dioxide, it is best to use an apparatus of the type to be described later or to remove the excess of the interfering constituent by precooling and filtering the mixture to be extracted.

The larger apparatus must be thoroughly dry before beginning operations in order to avoid the formation of ice in the system. It is best to make a practice of washing the condenser and column with acetone and drying with dry air before cooling the apparatus.

The cooling jacket, $D$, is now filled with a thick slush of solid carbon dioxide in a mixture of chloroform and carbon tetrachloride and the jacket, $L$, is cooled to $-40^{\circ}$ or $-50^{\circ} \mathrm{C}$. by gradual addition of solid carbon dioxide to the cryostat liquid. The jacket, $L$, is maintained at the higher temperature because the sulphur dioxide which collects in the column would freeze at the sublimation temperature of carbon dioxide. The lower temperature can be used in $D$, since the sulphur dioxide drains from the condenser before it reaches the freezing temperature. It is well also, as a precaution, to connect all vents, such as $E_{1}, E_{2}, E_{3}, E_{4}$, and $F$, to a cold trap by rubber tubes to prevent the escape of sulphur dioxide into the room.

The stopcocks are greased lightly with an ordinary stopcock grease and the keys inserted firmly after the apparatus has cooled. To fill the apparatus, stopcock $P$ is closed, $Q$, is opened to $R_{3}$ or $R_{4}$, and the sulphur dioxide distilled in through $K$ until about $100 \mathrm{ml}$ runs down into the boiler, or chilled liquid sulphur dioxide may be poured in through $F$. Should the sulphur dioxide be a residue from a previous operation,

it may be conveniently separated at this stage of the process from 
any immiscible hydrocarbon by liquefying and pouring it into a cold separatory funnel from which the lower layer can be drained into the column. In this case stopcock $P$ is left open long enough to allow all the sulphur dioxide which may be contaminated with hydrocarbon to run down into the boiler. The sulphur dioxide can be purified then by distillation through the apparatus, and the nonvolatile residue drained from the boiler.

The hydrocarbon is placed in $R_{1}$, with stopcock $B$ closed and about a 3 or 4 pound pressure (air, $\mathrm{CO}_{2}, \mathrm{~N}_{2}$ ) impressed on it through $A$. The circulation of sulphur dioxide is begun by starting the heater under the boiler which contains the sulphur dioxide and bringing the bath surrounding the column to the desired temperature.

The hydrocarbon is admitted slowly by opening stopcock, $B$. It has been found much easier to regulate the flow by this stopcock than by the gas pressure. If the hydrocarbon refuses to flow in, it is probable that the ice has formed in the inlet tube because the apparatus was not completely dried. In such event the apparatus must be emptied and brought up to room temperature again. In any case a short time is required to build up an immiscible layer above the sulphur dioxide in the column before liquid begins to run into receiver $R_{2}$. As long as the operation of extraction is continued it is only necessary to change the stopcock, $Q$, and the heater, and to drain out the degassed extract when the sulphur dioxide is driven out of a boiler. A small quantity of sulphur dioxide is unavoidably carried along with the product, so that it may be necessary to introduce fresh sulphur dioxide occasionally. Experience has shown that the operating capacity of the apparatus described is about one-half liter of hydrocarbon per hour.

As soon as the last of the hydrocarbon has run in, the gas pressure at $A$ must be cut off to prevent sulphur dioxide from being blown out of the column.

The procedure to be employed in emptying the apparatus will depend on whether the operation of extraction is finished or merely temporarily suspended. If it is finished, the hydrocarbon in the column should be separated from the sulphur dioxide. This may be done by performing one or two cycles of sulphur dioxide circulation after the flow of hydrocarbon has been stopped. In this way most of the sulphur dioxide which contains hydrocarbon is washed from the column. Then stopcock $P$ is opened to empty the column into one of the boilers and the sulphur dioxide is distilled off into the other boiler. The residue will contain the floating immiscible layer and some extracted material.

To store the sulphur dioxide it can be run directly from the boiler into a chilled "1-pound sulphur dioxide sample bottle" from which the valve has been temporarily removed. When the apparatus is to be filled again this cylinder can be cooled and the contents poured directly into the column through $F$ or it may be distilled from the warm cylinder as described above.

If the extraction is merely suspended or if it is desired to stop the operation quickly, the column may be drained into one of the boilers $111260-32-4$ 
and the mixture run into a chilled cylinder and sealed. The sulphur dioxide and hydrocarbon may then be separated at leisure by distillation, or the cylinder may be again cooled and the immiscible material separated in a cold separatory funnel as mentioned above. Sulphur dioxide which has been used in the extraction of even moderately high boiling hydrocarbons $\left(150^{\circ} \mathrm{C}\right.$.) will contain a considerable amount of the hydrocarbon after one distillation without rectification, so that care must be taken if it is to be used subsequently where contamination is undesirable.

\section{CONSTRUCTION OF METAL APPARATUS}

By using metal is it possible to construct an apparatus which will function at pressures of several atmospheres and will therefore permit the use of solvents considerably above their boiling points. This makes it possible to extract materials which will congeal or become very viscous at the temperatures at which the open type must be operated. The change in the relative miscibility of the constituents with the temperature may also be investigated over a much wider range than with the glass apparatus. ${ }^{10}$

The chief difference in the construction of the two types lies in the use of pressure equalizing connections between various parts of the apparatus instead of vents to the atmosphere.

Figure 3 is a diagram of an apparatus which has been operated at $+13^{\circ} \mathrm{C}$. The barrel of the column, $G_{3}$, is made of three-fourths inch iron pipe about 30 inches long with sections of one-fourth inch pipe, $G_{1}$, placed at either end. The enlargements, $G_{2}$, are formed by inserting pipe couplings at these points. This decreases the velocity of counterflow at the points where liquid enters the column, thus preventing a dragging of the liquid in the wrong direction.

All connecting tubes in which liquid flows by gravity are made of three-sixteenths inch (outside diameter) copper tubing. Smaller tubes have too great a resistance to flow. All valves, except $B_{2}$, are of one-fourth inch bore for the same reason. The tubes $M_{1}$ and $M_{2}$ through which the gas distills are three-eighths inch (outside diameter) to allow rapid flow of gas. The pressure-equalizing tubes, $E$, are of small diameter. The needle valve, $B_{2}$, permits close regulation of flow in the inlet tube.

The sight glasses, of which a detail is given in Figure 4, are of soldered pyrex-to-copper construction, which requires no packing. Packed sight glasses obtainable on the market may be satisfactory, however. Glasses $F_{1}, F_{2}$, and $F_{4}^{\prime}$ are about $4 \mathrm{~mm}$ in bore to allow easy flow of liquid. Glass $F_{3}$ is of smaller bore (about $1 \mathrm{~mm}$ ) to make the slow flow of liquid in the inlet line easily visible.

The reservoirs, $R_{1}, R_{2}, R_{3}$, and $R_{4}$ may be made from the iron cylinders in which sulphur dioxide is marketed in 6-pound quantities. If the heaters are to be operated at 110 volts and are to be used for sulphur dioxide, $R_{3}$ and $R_{4}$ should be wound with about 18 feet and $R_{2}$ with 24 feet of No. $24 \mathrm{~B}$. \& S. gage Chromel wire. The coils may be insulated from the cylinders with sheet mica and covered with asbestos. Valves $B_{7}$ and $B_{8}$ should be situated as far as possible from the boilers to keep the packing from melting.

10 It has been found by Moore, Morrell, and Egloff (Ind. Eng. Chem., vol. 18, p. 396, 1918) that the miscibility of cyclic hydrocarbons in sulphur dioxide increases much more rapidly with a rise in temperature than that of the straight-chain hydrocarbons. 


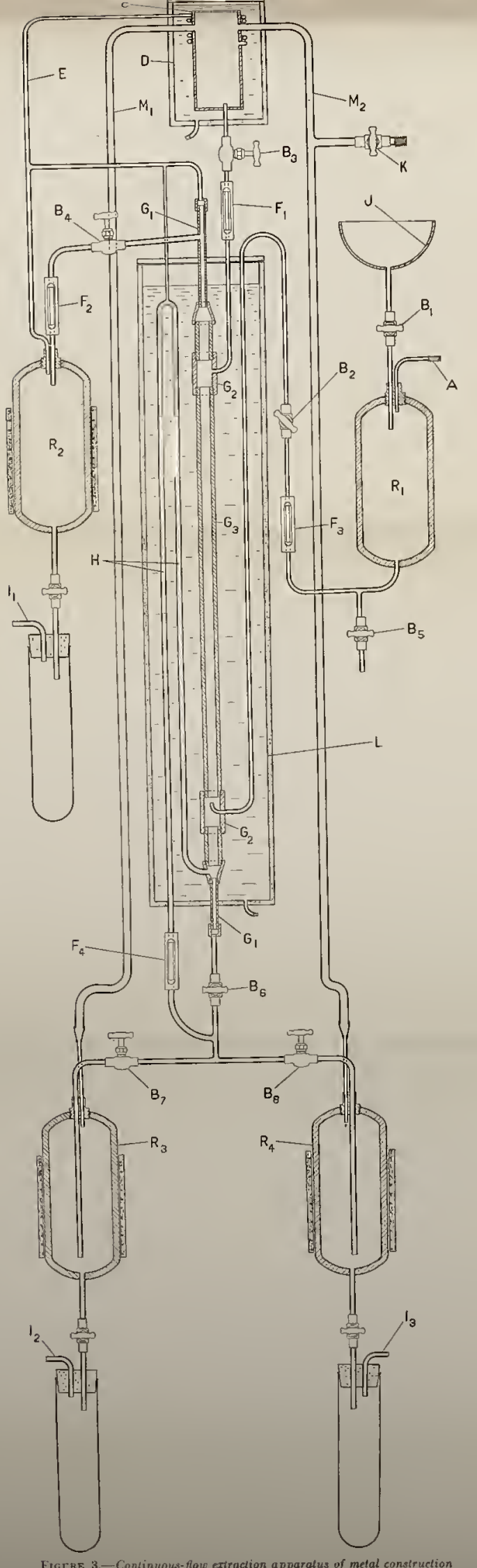

FtGCRE 3.-Continuots-flow extraction apparatus of metal construction 
The apparatus is mounted compactly in a frame consisting of four three-eighths inch iron pipes in 6-inch square formation.

\section{OPERATION OF THE METAL APPARATUS}

Precaution in the matter of drying this apparatus is unnecessary because of its closed construction. Before filling with sulphur dioxide all valves to the atmosphere should be closed, as well as $B_{2}, B_{4}, B_{6}$, and either $B_{7}$ or $B_{8}$. The condenser, $C$, and the column are then surrounded by a cooling agent, such as ice, ice salt, or carbon dioxide slush, depending on the temperature of extraction desired. If a thick slush of carbon dioxide ice is used on the condenser, valve $B_{3}$ must be opened wide and care should be exercised not to surround the column with a bath which will freeze the sulphur dioxide.

The sulphur dioxide container is connected to $K$ by means of a copper tube and small union. The sulphur dioxide may then be distilled in, or if the container is a small tank it may be mounted in an inverted position and the sulphur dioxide forced in as a liquid by slightly warming the tank.

After filling, it is unnecessary to remove the sulphur dioxide, except for repairing or cleaning the apparatus. The operation of removal can be most easily performed by cooling the condenser, $C$, with carbon dioxide slush leaving valves $B_{3}, B_{6}$, and either $B_{7}$ or $B_{8}$ open. (It is unnecessary to cool the column for the operation.) This will cause the sulphur dioxide in the extract boiler to be cooled below its atmospheric boiling point by evaporation. It can then be run directly into a cooled metal cylinder as described above. All such operations are much more pleasant if precautions are taken to connect the exits to the atmosphere to carbon dioxide-slush traps or absorbers. A piece of cotton soaked in ammonia water can be used to detect small leaks in the apparatus.

Before introducing the hydrocarbon into $R_{1}$, it is well to open $B_{5}$ momentarily to be sure that the reservoir is empty. The introduction of the hydrocarbon can be accelerated by applying a slight suction at $A . \quad B_{1}$ is then closed and $A$ is connected to a source of gas pressure through a reducing valve.

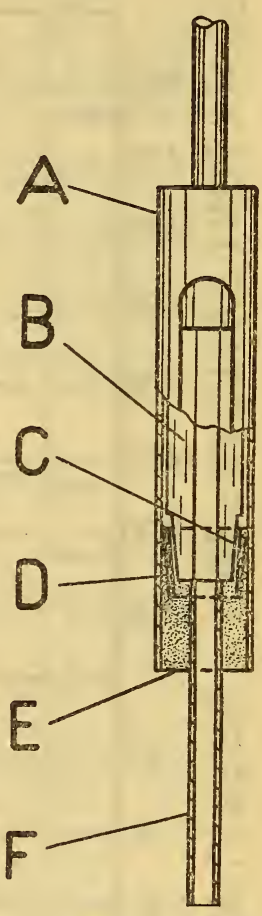

FIGURE 4.-Sight glass detail

$A$, copper or brass protecting sheath; $B$, heavy wall Pyrex laboratory glass tube tapered and ground into a copper ring $D$ at each end; $C$, soldered joint between glass taperand copper ring; $D$, copper ring tinned with solder inside and out; $E$, solder cast into sheath, $A$, holding $D$ and $F$ in position; $F$, copper tube. The pressure required will depend on the vapor pressure of sulphur dioxide at the temperatureof extraction.

The bath surrounding the column is next brought to the desired extraction temperature and the condenser bath is cooled to a somewhat lower temperature to increase the rate of condensation. If the condenser temperature is more than $20^{\circ}$ or $30^{\circ}$ lower than the column, however, there will be a tendency for sulphur dioxide to distil 
from the column and interfere with the flow of liquids. There will also be a large temperature difference between the contents of the column and the bath.

The circulation of sulphur dioxide is started by turning on the heater of the boiler in which the sulphur dioxide has collected. If the temperature of $C$ is above the freezing point of sulphur dioxide,

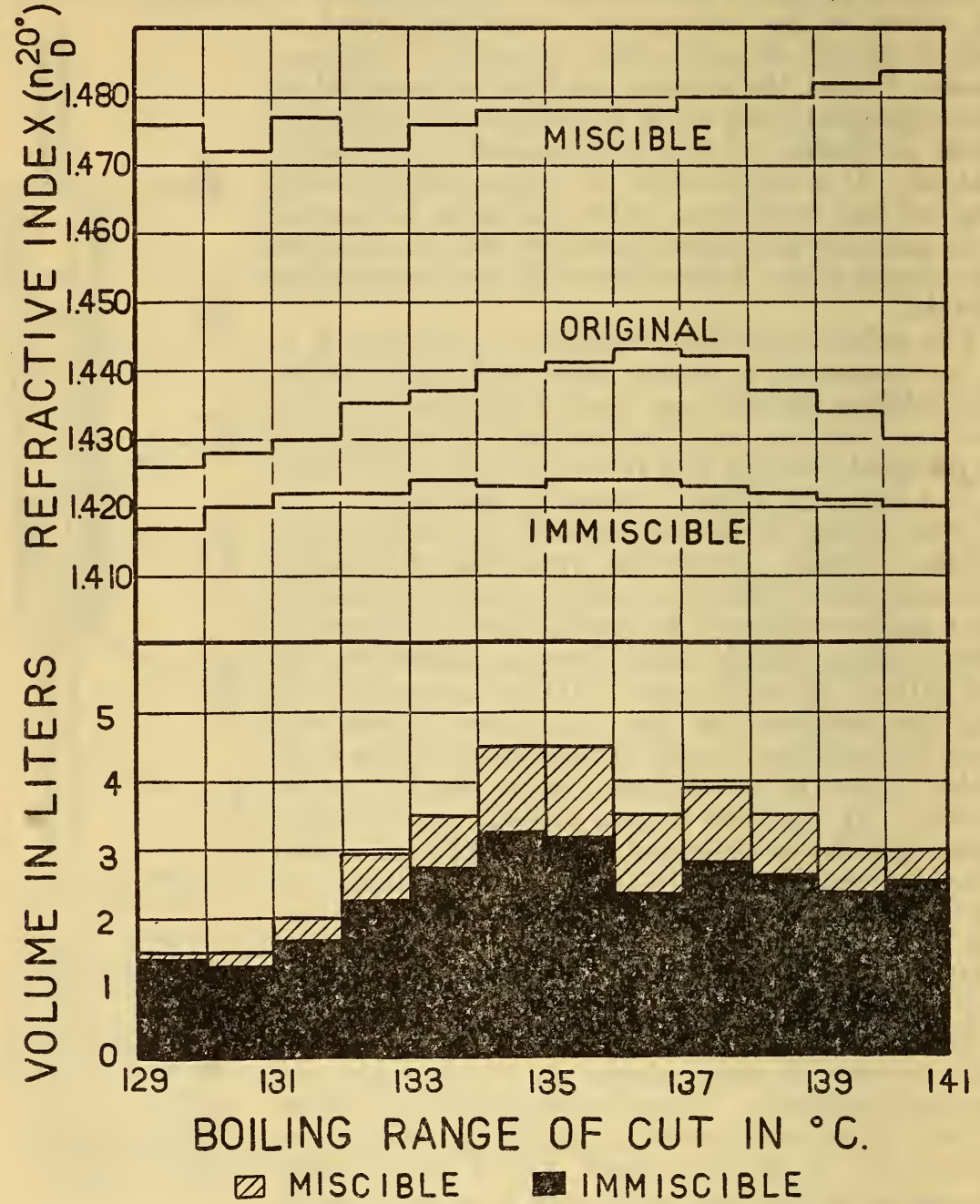

FIGURE 5.-Chart of an extraction of a fraction of petroleum

the flow may be regulated by $B_{3}$, otherwise it must be regulated by the heater.

Valve $B_{4}$ is then opened wide and $B_{2}$ opened slowly. An increased rate of flow of sulphur dioxide displaced by hydrocarbon will first appear in glass $F_{4}$ and in a short time a stream should appear in $F_{2}$. Observation is facilitated by placing a light behind the sight glasses.

It is possible to determine when all of the sulphur dioxide has been driven from a boiler by occasionally feeling the bottom. A sudden 
increase of temperature occurs when the sulphur dioxide has been driven out. When this rise is observed the extracted hydrocarbon is blown out into a flask or test tube as shown in Figure 3, and the cycle reversed. The ejected hydrocarbon will contain some sulphur dioxide especially if the extraction is done at higher temperatures. Therefore it is necessary to introduce fresh sulphur dioxide into the apparatus occasionally. In order to prevent the escape of sulphur dioxide into the room, the receivers should be connected to solid carbon dioxide traps through $I_{1}, I_{2}$, and $I_{3}$. The heater on $R_{2}$ aids in degassing the immiscible material before it is expelled, but low boiling liquids should be kept at a low temperature or fractional distillation will occur.

$B_{2}$ should be closed as soon as the last of the hydrocarbon has run in, otherwise a pressure of inert gas, which will interfere with distillation, may be built up in the apparatus. The contents of the column are then drained into one of the boilers by opening $B_{6}$, and the sulphur dioxide distilled off into the other boiler. Several distillations made in this way also serve to purify the sulphur dioxide for subsequent use.

The apparatus as described has been found to operate satisfactorily at a rate of 2 liters of hydrocarbon per hour.

\section{PERFORMANCE OF THE APPARATUS}

The glass apparatus has been used in the extraction of about 40 liters of petroleum fraction boiling in the range of temperature which includes the xylenes and other aromatic hydrocarbons in addition to a large proportion of paraffinic material. ${ }^{11}$ Figure 5 is a graphical presentation of results obtainable with the apparatus when operated as described. Since the refractive indices of the aromatic compounds are much higher than those of the paraffins, the difference in refractive index of the miscible and immiscible fractions is indicative of the efficiency of extractions. ${ }^{12}$ Further extraction of the immiscible material was found to change the refractive index very little.

The metal apparatus has been thoroughly tested with a sample of high-test gasoline containing considerable aromatic material and found to work smoothly. An attempt was also made to remove cyclic hydrocarbons from a petroleum fraction boiling in the neighborhood of $100^{\circ} \mathrm{C}$. Extraction was tried at temperatures ranging from -10 to $+13^{\circ}$ with little evidence of separation, however.

\section{ACKNOWLEDGMENT}

The author acknowledges the technical advice of $\mathrm{E}$. W. Washburn director of the American Petroleum Institute project No. 6, and also that of J. D. White and F. W. Rose.

Washington, February 13, 1932.

11 Used by J. D. White and F. W. Rose, jr., as a step in a process for separating xylenes from petroleum. 12 Unpublished data of J. D. White and F. W. Rose, Jr. 